\title{
BMJ Open Diabetes Shared Care Program (DSCP) and risk of infection mortality: a nationwide cohort study using administrative claims data in Taiwan
}

\author{
Cheng-Han Chen, ${ }^{1,2}$ Sheng-Hsiang Ma, ${ }^{2,3}$ Sung-Yuan Hu, ${ }^{4,5}$ Chia-Ming Chang, ${ }^{1,2,6}$ \\ Jen-Huai Chiang, ${ }^{7}$ Vivian Chia-Rong Hsieh, ${ }^{8}$ David Hung-Tsang Yen, ${ }^{1,2}$ \\ Chorng-Kuang How, ${ }^{1,2}$ Ming-Shun Hsieh ${ }^{1,2,6,9}$
}

To cite: Chen C-H, Ma S-H, Hu S-Y, et al. Diabetes Shared Care Program (DSCP) and risk of infection mortality: a nationwide cohort study using administrative claims data in Taiwan. BMJ Open 2018;8:e21382. doi:10.1136/ bmjopen-2017-021382

- Prepublication history for this paper is available online. To view these files, please visit the journal online (http://dx.doi org/10.1136/bmjopen-2017021382).

$\mathrm{C}-\mathrm{KH}$ and M-SH contributed equally.

Received 3 January 2018

Revised 14 May 2018

Accepted 16 May 2018

\section{Check for updates}

For numbered affiliations see end of article.

Correspondence to Dr Ming-Shun Hsieh; simpleabei@yahoo.com.tw

\section{ABSTRACT}

Objective The Diabetes Shared Care Program (DSCP) is an integrated care model in Taiwan that has been proven to improve the care quality of patients with diabetes. We aimed to evaluate the efficacy of DSCP in decreasing the hospital mortality of infectious diseases.

Methods From 1662929 patients with type 2 diabetes newly diagnosed between 1999 and 2013, we retrieved a total of 919 patients who participated in the DSCP with the first hospitalisation for an infectious disease as the study cohort and 9190 propensity score-matched patients with type 2 diabetes who did not participate as the comparison. The efficacy of DSCP was evaluated via the following comparisons between the DSCP and non-DSCP cohorts: hospital mortality, 1-year medical cost prior to and during the hospitalisation, and complications, such as receiving mechanical ventilation and intensive care unit admission. The ratio (OR) for hospital mortality of the DSCP participants was calculated by logistical regression. Further stratification analyses were conducted to examine which group of patients with type 2 diabetes benefited the most from the DSCP during hospitalisation for infectious diseases.

Results The DSCP cohort had a lower hospital mortality rate than the non-DSCP participants $(2.18 \%$ vs $4.82 \%$, $\mathrm{p}<0.001)$. The total medical cost during the hospitalisation was lower in the DSCP cohort than in the non-DSCP cohort (NT\$72 454 \pm 30429 vs NT\$86 385 \pm 29350$)(p=0.006)$. In the logistical regression model, the DSCP participants exhibited a significantly decreased adjusted $\mathrm{OR}$ for hospital mortality (adjusted $\mathrm{OR}=0.42,95 \% \mathrm{Cl} 0.26$ to 0.66 , $p=0.0002$ ). The efficacy of the DSCP was much more prominent in male patients with type 2 diabetes and in patients with lower incomes.

Conclusion Participation in the DSCP was associated with a lower risk of hospital mortality for infectious diseases.

\section{INTRODUCTION}

Type 2 diabetes mellitus (DM) is an important healthcare issue in both low/middle-income countries and developed countries, with a reported prevalence of $9 \%$ in Caucasians and approximately 10\%-20\% among other
Strengths and limitations of this study

- This study was conducted using the nationwide claims database of 1662929 patients with type 2 diabetes newly diagnosed between 1999 and 2013 to evaluate the efficacy of the Diabetes Shared Care Program (DSCP) in decreasing the hospital mortality of infectious diseases.

- All the DSCP participants were enrolled for at least 1 year to evaluate the efficacy by assessing medical cost and hospital outcomes.

- Propensity score matching was conducted in this current study to reduce the selection bias between the DSCP and non-DSCP cohorts. Stratification analyses were conducted to examine which group of participants benefited the most from the DSCP.

- The limitation of this study was its observational and retrospective design, thereby making it impossible to randomly assign the DSCP enrollees.

- No laboratory data were available in this current study since this nationwide database was originally designed for expenditure claims.

races. ${ }^{12}$ In Taiwan, the prevalence of type $2 \mathrm{DM}$ is approximately $6 \% .{ }^{3}$ Recently, the prevalence of type 2 diabetes has risen worldwide. According to National Health Interview Surveys conducted between 1999 and 2009 in the USA, there was a $9 \%$ increase in patients with diabetes. ${ }^{45}$

Obesity, one of the most well-established risk factors of diabetes, was on the rise in Taiwan. Approximately one-fourth of adults in Taiwan were obese and therefore prone to develop type 2 diabetes. ${ }^{3}{ }^{6}$ The numbers of patients with type 2 diabetes with comorbidities of hypertension and hyperlipidaemia also increased considerably from $10.47 \%$ in 2000 to $25.65 \%$ in $2009 .^{5} 7$ The increasing number of patients with type 2 diabetes places a considerable burden on the healthcare system in Taiwan; the medical cost of 
the population increased from $3 \%$ to $4.5 \%$ of the total Taiwan's National Health Insurance (NHI) programme expenditures from 1986 to $2009 .^{8}$

If left uncontrolled, type 2 diabetes would induce many detrimental complications, such as diabetic nephropathy, neuropathy, retinopathy, peripheral arterial occlusive disease and cardiovascular disease. ${ }^{19} 10$ Infectious diseases are an important complication in patients with type 2 diabetes. A Danish cohort study demonstrated that patients with type 2 diabetes were at an increased risk for hospitalisation related to infection. ${ }^{11}$ Therefore, making lifestyle modifications and receiving medical treatments after the diagnosis of type 2 diabetes is important.

In 2001, the Ministry of Health and Welfare in Taiwan initiated the Diabetes Shared Care Program (DSCP) to help patients with type 2 diabetes achieve better glycaemic control. ${ }^{1}$ This care model emphasised the team care of the treating physicians, diabetes specialists, nurses and dietitians with the aim of improving the quality of care for patients with diabetes. ${ }^{9}$ Previous studies have demonstrated the improvement of cardiovascular and metabolic outcomes in the DSCP participants of patients with type 2 diabetes. ${ }^{9}{ }^{12}$ However, currently, no studies have investigated the influence of the DSCP on the outcomes of infection-related hospitalisation. In this study, we aimed to evaluate the efficacy of the DSCP in patients with type 2 diabetes hospitalised for infectious diseases.

\section{METHODS}

Study population

This observational, retrospective cohort study was conducted using the Longitudinal Cohort of Diabetes Patients (LHDB) data set, which was obtained from Taiwan's NHI programme. Taiwan's NHI programme is a universal health insurance system that covers nearly $99 \%$ of its residents. ${ }^{13}$

This study retrieved patients aged between 18 and 100 years old who were newly diagnosed with type 2 diabetes and were first hospitalised for infectious conditions. From the 1662929 patients with diabetes newly diagnosed between 1999 and 2013 in the LHDB, 919 patients with diabetes participating in the DSCP with

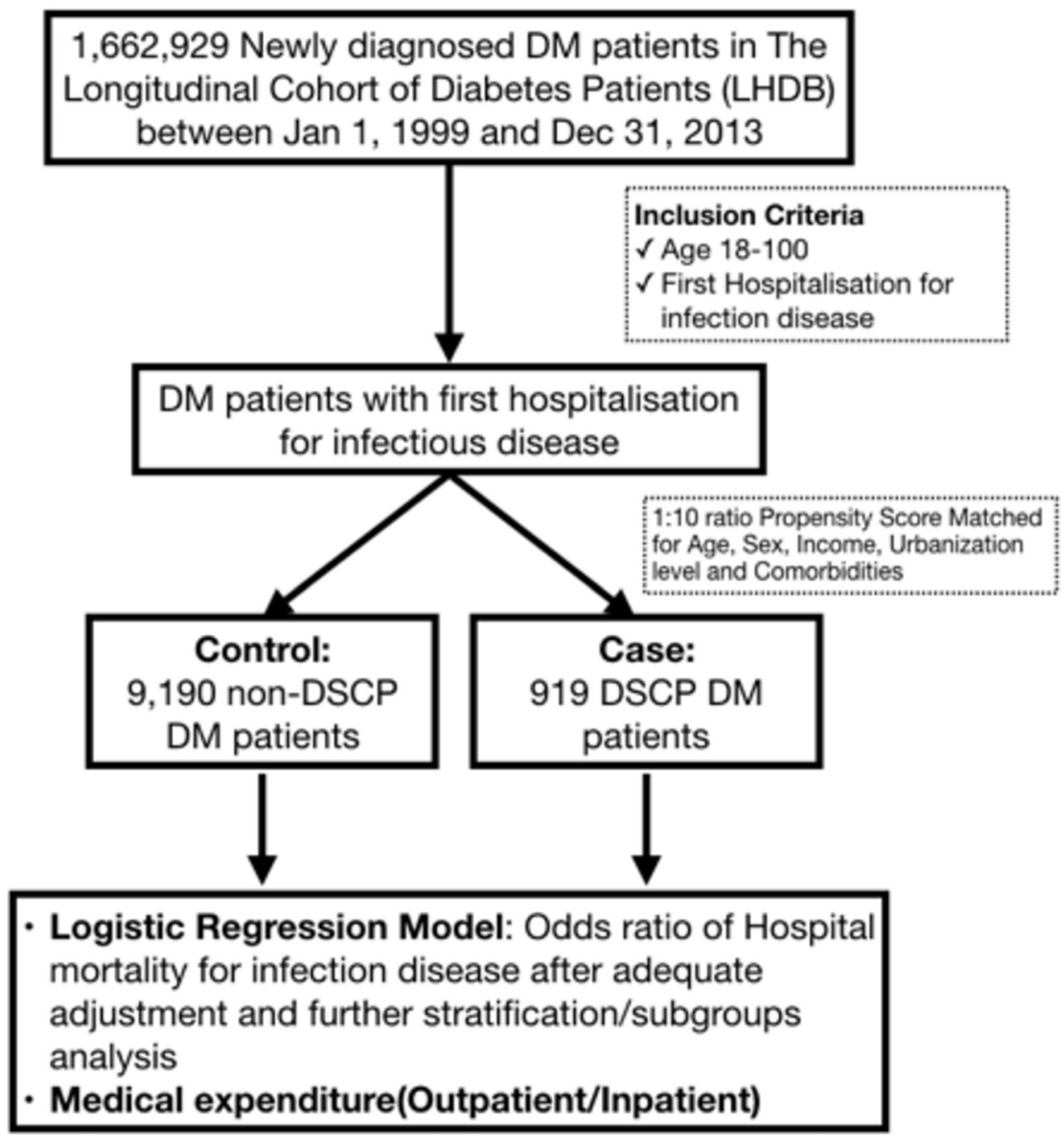

Figure 1 Participant selection process. DM, diabetes mellitus; DSCP, Diabetes Shared Care Program. 
the first hospitalisation for infection were retrieved (figure 1). Meanwhile, we conducted a 1:10 ratio propensity score match for patients who were not enrolled in the programme according to their age, sex, income, urbanisation level and comorbidities. ${ }^{14}{ }^{15}$ A total of 9190 propensity score-matched patients with diabetes who did not participate in the DSCP were assigned to the comparison group.

\section{Patient and public involvement}

Because the LHDB contains deidentified secondary data for research, our study was exempt from requiring informed consent from the participants. Thereby, no patient or public was involved in this study.

\section{Outcome evaluation}

This study aimed to evaluate whether the DSCP influenced the outcomes of infection-related hospitalisation in patients with diabetes. The index hospitalisation was defined as the first admission due to infectious disease after the patients participated in the DSCP. The primary outcome was the hospital mortality rate during the index hospitalisation. The secondary outcomes were the length of hospital stay, medical cost and complications between the DSCP and non-DSCP participants. We also analysed the difference in mortality ORs according to the infection sites between the DSCP and non-DSCP groups.

\section{Diabetes Shared Care Program}

The DSCP was initiated by Taiwan's Bureau of National Health Insurance (BNHI) in 2001 to enhance the quality and efficiency of type 2 diabetes care. ${ }^{13}$ This programme financially encourages the healthcare provider to provide patients with diabetes with multiple laboratory tests annually (eg, HbA1c, total cholesterol, low-density lipoprotein (LDL)) and other related health examinations (eg, for eyes or feet). ${ }^{13}$ Physicians specialising in metabolic disorders or who had taken part in a training programme for type 2 diabetes shared care were eligible to enrol individual patients. ${ }^{13}$ All patients enrolled were required to receive education and comprehensive care for type 2 diabetes by the BNHI. ${ }^{13}$ Therefore, DSCP participants received integrated care from physicians, diabetes educators and dietitians, whereas the non-DSCP participants received care from only physicians. However, the time between the enrolment date and index hospitalisation date was recorded.

\section{Statistics}

Descriptive analysis was used to compare the basic characteristics of the DSCP and non-DSCP groups. Demographic information, including age, sex, income, urbanisation level, comorbidities and medical resource utilisation, was collected retrospectively for a period of 1 year. A 1:10 ratio of propensity score-matched comparison was conducted based on the patient's age, sex, insurance premium, urbanisation level and comorbidities. ${ }^{14} 15$ The OR was calculated using the logistical regression model after adjusting for potential confounders to determine the effect of the DSCP on mortality in patients with diabetes hospitalised for infection. Further stratification analyses were conducted to examine which group of patients with type 2 diabetes benefited the most from the DSCP during hospitalisation for infectious diseases. Statistical analyses were performed using the SAS V.9.4 statistical package (SAS Institute). A p value of 0.05 was considered significant.

\section{RESULTS}

The demographic characteristics and comorbidities of patients with type 2 diabetes who did and did not participate in the DSCP are shown in table 1. The mean ages of the DSCP and non-DSCP participants were $61.77 \pm 13.91$ and $61.61 \pm 14.88$ years old, respectively $(p=0.74)$. There were no significant differences in the age, sex, income, urbanisation level or baseline comorbidities between each group. However, the DSCP participants were more likely to receive aspirin, statins, biguanide, sulfonylurea, thiazolidinedione and insulin.

The hospital outcomes during index hospitalisation for infection between the DSCP and non-DSCP participants are shown in table 2. In terms of the mortality rate during hospitalisation, the DSCP group showed a significantly lower rate $(2.18 \%$ vs $4.82 \%, p=0.0003)$. Stratification analysis of the different infectious diseases showed that the DSCP participants exhibited a lower frequency of respiratory (26.22\% vs $30.24 \%, \mathrm{p}=0.0112)$ and gastrointestinal infections $(8.49 \%$ vs $13.04 \%, \mathrm{p}<0.0001)$. In addition, primary bacteraemia $(12.4 \%$ vs $16.72 \%, \mathrm{p}=0.0007)$ and sepsis $(10.12 \%$ vs $14.67 \%, p=0.0002)$ were less frequent in the DSCP group. However, a higher frequency of genitourinary infections $(32.64 \%$ vs $28.96 \%, \mathrm{p}=0.019)$ and musculoskeletal system infections $(21.87 \%$ vs $16.72 \%$, $\mathrm{p}<0.0001)$ were reported in the DSCP group. Regarding the rate of mechanical ventilation requirement and intensive care unit admission, no significant differences were observed between the groups. The length of hospital stay was also not significantly different between the two groups.

The logistical regression model was conducted to measure the OR of hospital mortality associated with the DSCP in different infection classifications (table 3). For primary bacteraemia, the mortality rate was improved significantly in the DSCP group (adjusted $\mathrm{OR}=0.49,95 \%$ CI 0.20 to $0.66, p=0.0399$ ). Although no significant difference was noted, the mortality rates of respiratory infection (adjusted $\mathrm{OR}=0.5,95 \% \mathrm{CI} 0.25$ to $1.00, \mathrm{p}=0.051$ ) and sepsis (adjusted $\mathrm{OR}=0.55,95 \%$ CI 0.27 to $1.10, \mathrm{p}=0.0916$ ) were lower in the DSCP group.

The medical care expenditures in the DSCP and non-DSCP groups are shown in figure 2. The medical expenditures of the outpatient department during a 1-year period were significantly higher in the DSCP

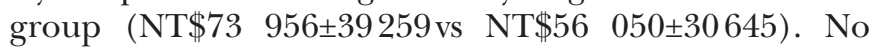
significant difference in the total medical expenditures 
Table 1 Demographic characteristics and comorbidities in patients with diabetes who did and did not participate in the

Diabetes Shared Care Program (DSCP)

\begin{tabular}{|c|c|c|c|c|c|}
\hline & \multicolumn{5}{|c|}{ After PS matching* } \\
\hline & \multicolumn{5}{|c|}{ Diabetes Shared Care Program } \\
\hline & \multicolumn{2}{|c|}{ No $(n=9190,90.91 \%)$} & \multicolumn{2}{|c|}{ Yes $(n=919,9.09 \%)$} & \multirow[b]{2}{*}{$P$ values } \\
\hline & $\mathbf{n}$ & $\%$ & $\mathbf{n}$ & $\%$ & \\
\hline Sex & & & & & 0.8242 \\
\hline Female & 3995 & 43.47 & 396 & 43.09 & \\
\hline Male & 5195 & 56.53 & 523 & 56.91 & \\
\hline Age (years) & & & & & 0.1122 \\
\hline $18-29$ & 176 & 1.92 & 10 & 1.09 & \\
\hline $30-39$ & 559 & 6.08 & 49 & 5.33 & \\
\hline $40-49$ & 1364 & 14.84 & 136 & 14.8 & \\
\hline $50-59$ & 2124 & 23.11 & 217 & 23.61 & \\
\hline $60-69$ & 1964 & 21.37 & 209 & 22.74 & \\
\hline $70-79$ & 1930 & 21 & 212 & 23.07 & \\
\hline$\geq 80$ & 1073 & 11.68 & 86 & 9.36 & \\
\hline Mean $( \pm \mathrm{SD}) \dagger$ & $61.61(14.88)$ & & $61.77(13.91)$ & & $0.7369 \dagger$ \\
\hline Insurance premium (NT\$) & & & & & 0.9102 \\
\hline$<20000$ & 4138 & 45.03 & 413 & 44.94 & \\
\hline $20000-40000$ & 3982 & 43.33 & 398 & 43.31 & \\
\hline $40000-60000$ & 882 & 9.6 & 86 & 9.36 & \\
\hline$\geq 60000$ & 188 & 2.05 & 22 & 2.39 & \\
\hline Urbanisation level & & & & & 0.9817 \\
\hline 1 (highest) & 1353 & 14.72 & 138 & 15.02 & \\
\hline 2 & 2770 & 30.14 & 270 & 29.38 & \\
\hline 3 & 2248 & 24.46 & 230 & 25.03 & \\
\hline 4 & 2010 & 21.87 & 198 & 21.55 & \\
\hline 5 (lowest) & 809 & 8.8 & 83 & 9.03 & \\
\hline \multicolumn{6}{|l|}{ Baseline comorbidity } \\
\hline HTN & 6500 & 70.73 & 652 & 70.95 & 0.89 \\
\hline Hyperlipidaemia & 4558 & 49.60 & 465 & 50.60 & 0.5628 \\
\hline COPD & 2841 & 30.91 & 285 & 31.01 & 0.9512 \\
\hline PAOD & 931 & 10.13 & 102 & 11.10 & 0.3554 \\
\hline IHD & 3254 & 35.41 & 332 & 36.13 & 0.6644 \\
\hline CLD & 2181 & 23.73 & 224 & 24.37 & 0.663 \\
\hline CKD & 3403 & 37.03 & 351 & 38.19 & 0.4861 \\
\hline Stroke & 2465 & 26.82 & 249 & 27.09 & 0.8592 \\
\hline Cancer & 1136 & 12.36 & 118 & 12.84 & 0.6746 \\
\hline CCl score & & & & & 0.1011 \\
\hline 0 & 190 & 2.07 & 18 & 1.96 & \\
\hline 1 & 1129 & 12.29 & 91 & 9.90 & \\
\hline$\geq 2$ & 7871 & 85.65 & 810 & 88.14 & \\
\hline \multicolumn{6}{|l|}{ Drug } \\
\hline NSAID & 3818 & 41.55 & 390 & 42.44 & 0.6008 \\
\hline Aspirin & 910 & 9.90 & 116 & 12.62 & 0.0092 \\
\hline Statin & 1728 & 18.80 & 212 & 23.07 & 0.0017 \\
\hline
\end{tabular}




\begin{tabular}{|c|c|c|c|c|c|}
\hline & \multicolumn{5}{|c|}{ After PS matching* } \\
\hline & \multicolumn{5}{|c|}{ Diabetes Shared Care Program } \\
\hline & \multicolumn{2}{|c|}{ No $(n=9190,90.91 \%)$} & \multicolumn{2}{|c|}{ Yes $(n=919,9.09 \%)$} & $P$ values \\
\hline Clopidogrel & 340 & 3.70 & 43 & 4.68 & 0.1382 \\
\hline Biguanides & 3081 & 33.53 & 438 & 47.66 & $<0.0001$ \\
\hline Sulfonylureas & 3556 & 38.69 & 533 & 58.00 & $<0.0001$ \\
\hline Thiazolidinedione & 548 & 5.96 & 105 & 11.43 & $<0.0001$ \\
\hline Other OAD & 955 & 10.39 & 173 & 18.82 & $<0.0001$ \\
\hline Insulin & 983 & 10.7 & 228 & 24.81 & $<0.0001$ \\
\hline
\end{tabular}

$\mathrm{X}^{2}$ test.

*Propensity score matching included the following variables: age, sex, insurance premium, urbanisation and baseline comorbidities.

†Two-sample t-test.

†Fisher's exact test.

§Antidiabetic agents include biguanides, DPP-4 inhibitors, other OAD, sulfonylureas, thiazolidinedione and insulin.

$\mathrm{CCl}$, Charlson Comorbidity Index; CKD, chronic kidney disease; CLD, chronic liver disease; COPD, chronic obstructive pulmonary disease; HTN, hypertension; IHD, ischaemic heart disease; NSAID, non-steroidal anti-inflammatory drug; OAD, oral antidiabetic drug; PAOD, peripheral arterial occlusion disease; PS, propensity score.

before the index hospitalisation between the two groups was noted (NT\$182 988 \pm 233434 vs NT\$176 253 \pm 314730$)$ $(\mathrm{p}=0.4212)$. However, the total medical expenditures during the index hospitalisation were significantly higher in the non-DSCP group (NT\$72 454 \pm 30429 vs NT $\$ 86$ $385 \pm 29350) \quad(\mathrm{p}=0.0066)$.

Table 2 Hospital outcomes during index hospitalisation for infectious disease between patients with diabetes who did and did not participate in the DSCP

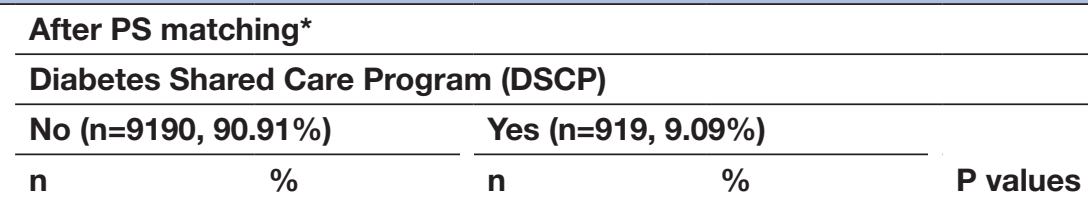

\begin{tabular}{|c|c|c|c|c|c|}
\hline \multicolumn{6}{|l|}{ Infection classification } \\
\hline Central nervous & 48 & 0.52 & 7 & 0.76 & 0.3469 \\
\hline Respiratory & 2779 & 30.24 & 241 & 26.22 & 0.0112 \\
\hline Gastrointestinal & 1198 & 13.04 & 78 & 8.49 & $<0.0001$ \\
\hline Genitourinary & 2661 & 28.96 & 300 & 32.64 & 0.0191 \\
\hline Primary bacteraemia & 1537 & 16.72 & 114 & 12.4 & 0.0007 \\
\hline Device-related infection & 138 & 1.5 & 15 & 1.63 & 0.7572 \\
\hline Other/undetermined & 1039 & 11.31 & 130 & 14.15 & 0.0103 \\
\hline Sepsis & 1348 & 14.67 & 93 & 10.12 & 0.0002 \\
\hline \multicolumn{6}{|l|}{ Procedures (during hospitalisation) } \\
\hline Mortality & 443 & 4.82 & 20 & 2.18 & 0.0003 \\
\hline Length of hospital stay, days (means, SD) & \multicolumn{2}{|c|}{$11.76(29.24)$} & \multicolumn{2}{|c|}{$11.36(11.78)$} & $0.4203 \ddagger$ \\
\hline
\end{tabular}

$\mathrm{X}^{2}$ test.

*Propensity score matching included the following variables: age, sex, insurance premium, urbanisation and baseline comorbidities.

†Fisher's exact test.

$\ddagger$ Two-sample t-test.

PS, propensity score. 
Table 3 Logistical regression model measuring the ORs and 95\% Cls of mortality associated with the Diabetes Shared Care Program stratified by infection classification

\begin{tabular}{|c|c|c|c|c|c|c|}
\hline \multirow[b]{2}{*}{ Variable } & \multicolumn{2}{|l|}{ Mortality } & \multicolumn{2}{|l|}{ Crude } & \multicolumn{2}{|l|}{ Adjusted* } \\
\hline & No n (\%) & Yes n (\%) & OR $(95 \% \mathrm{Cl})$ & $P$ values & OR $(95 \% \mathrm{Cl})$ & $P$ values \\
\hline \multicolumn{7}{|l|}{ Infection classification } \\
\hline Central nervous & $6(12.50)$ & $2(28.57)$ & $2.8(0.44$ to 17.80$)$ & 0.2752 & - & - \\
\hline Respiratory & $190(6.84)$ & $9(3.73)$ & $0.53(0.27$ to 1.05$)$ & 0.0669 & 0.5 (0.25 to 1.00$)$ & 0.051 \\
\hline Cardiovascular & 0 & 0 & - & - & - & - \\
\hline Gastrointestinal & $34(2.84)$ & $2(2.56)$ & 0.9 (0.21 to 3.82$)$ & 0.8875 & 1.06 (0.24 to 4.74$)$ & 0.9433 \\
\hline Genitourinary & $50(1.88)$ & 0 & - & - & - & - \\
\hline Musculoskeletal & $10(0.65)$ & $1(0.50)$ & 0.76 (0.10 to 6.00$)$ & 0.7977 & 0.67 (0.08 to 6.01$)$ & 0.7212 \\
\hline Primary bacteraemia & $273(17.76)$ & $10(8.77)$ & 0.45 (0.23 to 0.86$)$ & 0.0166 & $0.49(0.25$ to 0.97$)$ & 0.0399 \\
\hline Device related & $5(3.62)$ & 0 & - & - & - & - \\
\hline Other/undetermined & $17(1.64)$ & $1(0.77)$ & 0.47 (0.06 to 3.53$)$ & 0.46 & 0.42 (0.05 to 3.42) & 0.4165 \\
\hline Sepsis & 267 (19.81) & $10(10.75)$ & 0.49 (0.25 to 0.95$)$ & 0.0356 & 0.55 (0.27 to 1.10$)$ & 0.0916 \\
\hline
\end{tabular}

*Adjusted for age, sex, insurance premium, urbanisation level and comorbidities in a logistical regression model.

The forest plot in figure 3 shows the stratification of ORs of the DSCP efficacy. In our stratification analysis, men participating in the DSCP had a lower OR for mortality rate (adjusted $\mathrm{OR}=0.36$ ) compared with women. Therefore, men may have benefited more from this programme. The efficacy of DSCP in infectious diseases was much more obvious in male patients (adjusted $\mathrm{OR}=0.36,95 \%$ CI 0.20 to $0.66, \mathrm{p}=0.0009$ ), patients aged $70-79$ years (adjusted $\mathrm{OR}=0.25,95 \%$ CI 0.09 to $0.70, \mathrm{p}=0.008$ ) and patients with the low and lowest incomes (adjusted $\mathrm{OR}=0.32$, 95\% CI 0.12 to $0.87, \mathrm{p}=0.0256$ and adjusted $\mathrm{OR}=0.41,95 \%$ CI 0.23 to $0.73, \mathrm{p}=0.0022$, respectively).

\section{DISCUSSION}

Our study demonstrated that the patients with type 2 diabetes enrolled in the DSCP were associated with better outcomes from infection-related hospitalisation. Among

$$
P=0.4212
$$

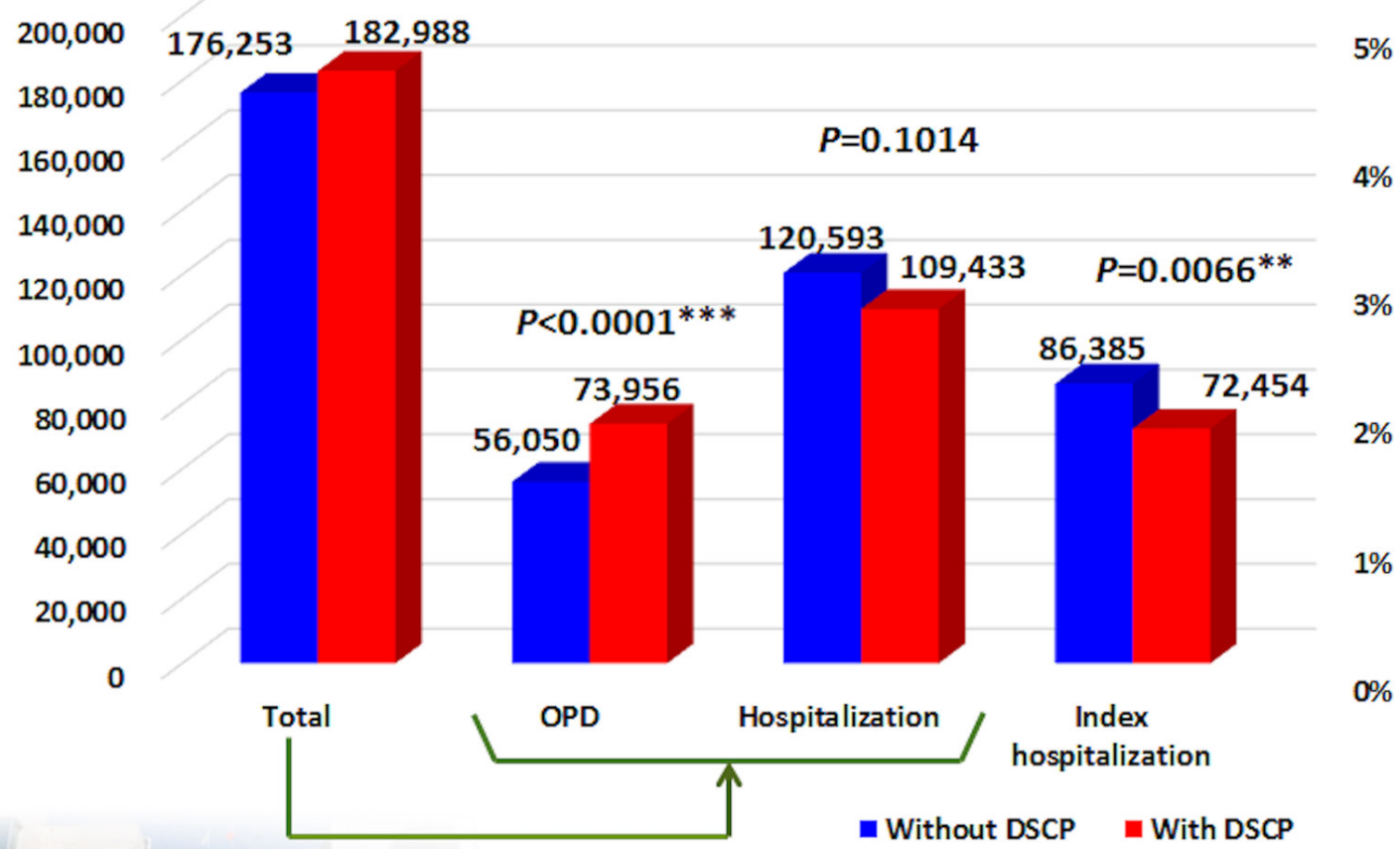

$P=0.0003^{* * *}$

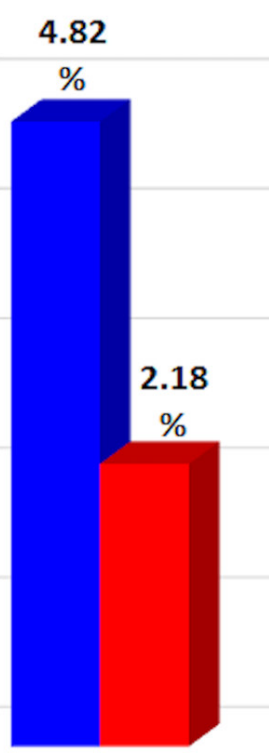

Mortality

Figure 2 Cost and mortality rate in patients with diabetes with and without DSCP participation. DSCP, Diabetes Shared Care Program; OPD, outpatient department. 


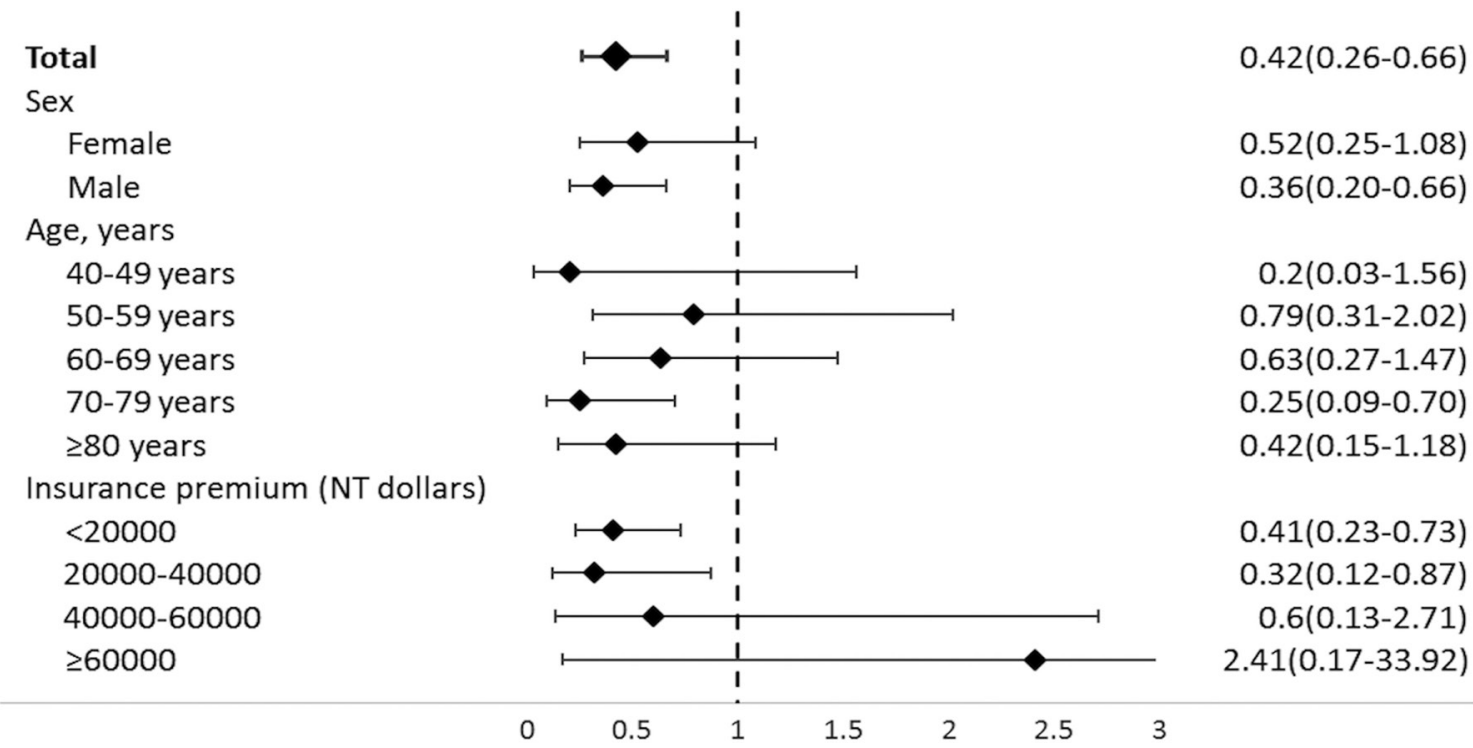

Figure 3 Stratification analysis of Diabetes Shared Care Program (DSCP) efficacy in infectious conditions with hospitalisation.

patients enrolled in the DSCP, the in-hospital mortality rate was significantly lower than that of patients not enrolled in the DSCP $(2.18 \%$ vs $4.82 \%, \mathrm{p}=0.0003)$. The total medical expenditures during the index hospitalisation were significantly lower in the DSCP group (NT\$72 $454 \pm 30429$ vs NT $\$ 86385 \pm 29350, \mathrm{p}=0.0066$ ). Even without statistical significance, the DSCP group had lower requirements of mechanical ventilation support and intensive care unit admission. The medical expenditure of index hospitalisation was also significantly lower in the DSCP group (NT\$72 454 \pm 30429 vs NT\$86 $385 \pm 29350$, which are equal to US $\$ 2468.70$ vs US $\$ 2944.02$ ). A previous study also reported that the DSCP increased the total expenses in type 2 diabetic care to approximately US $\$ 100$ (equal to NT\$3000) per person per year, which indeed lowers the costs of hospitalisation. ${ }^{13}$ In stratification analysis of DSCP efficacy in infectious conditions with hospitalisation, male patients, patients aged between 70 and 79 years and patients with insurance premiums below NT $\$ 40000$ (equal to US\$1333.33) benefited more from the DSCP.

Pay for performance with the DSCP was established in 2001 in Taiwan and has proven to be cost-effective, especially in patients with type 2 diabetes with hypertension and hyperlipidaemia. ${ }^{158}$ The programme was first launched in August 1996 in I-Lan County, which is located in northeastern Taiwan. ${ }^{1}$ The DSCP was then implemented nationwide in Taiwan. The number of patients participating in this programme has increased gradually since $2001 .^{112}$ This programme aims to provide comprehensive type 2 diabetic care by integrating physical and psychosocial support. The DSCP sets an 'ABC' goal for diabetic management (A: HbA1c $<7.0 \%$, B: blood pressure $<130 / 80 \mathrm{~mm} \mathrm{Hg}$, C: LDL cholesterol $<100 \mathrm{mg} /$ $\mathrm{dL} /$ total cholesterol $<160 \mathrm{mg} / \mathrm{dL}) .{ }^{1}$ This programme also encourages healthcare providers with financial incentives to perform regular follow-up visits and exams for better monitoring and controlling diabetes. ${ }^{13}$ In terms of medication, the classes of antidiabetic, antihypertensive and antilipidemic medications were prescribed individually according to the patient's care plan, comorbidity and other patient factors. ${ }^{9}$ In addition to medical treatments, patients who participate in the DSCP are educated about self-care, nutrition supplements and other health issues related to diabetes by certificated diabetes specialists, nurses and dietitians. ${ }^{9}$ Chen et al ${ }^{16}$ illustrated that the duration of enrolment in this programme was positively correlated with the care quality and compliance. ${ }^{16}$ Patients with type 2 diabetes with 1 year of participation in the DSCP also benefited more than those participating fewer than 3 months. ${ }^{816}$

Hao et at found that patients enrolled in the DSCP had significantly improved systolic and diastolic blood pressure. Lipid profiles, including high-density lipoprotein and LDL, showed significant improvements after participation in the DSCP for 3 years. ${ }^{9}$ Kornelius $e t ~ a l^{12}$ also reported that patients engaged in the DSCP had a $14 \%$ reduction in cardiovascular events, a $16 \%$ reduction in stroke risk and a $22 \%$ reduction in all-cause mortality. These authors also found that patients with lower incomes and those of older age were at higher risk of cardiovascular complications. ${ }^{12}$

In addition, the immune system is compromised in patients with diabetes, and these patients are more likely to develop infectious diseases. Previous studies have reported that diabetes may lead to deleterious effects on both the cellular and humoral immune system, causing patients with diabetes to be more susceptible to infection. ${ }^{17}{ }^{18}$ In terms of pathophysiology, decreased perception, tissue hypoxaemia, reduced tissue perfusion and impaired leucocyte function develop in patients with diabetes. ${ }^{19}$ These changes also include decreased leucocyte function, altered microvascular responses 
and changes in the complement cascade and cytokine network. ${ }^{1718}$ Furthermore, leucocyte responsiveness in patients with diabetes has been proven to be inversely related to the hyperglycaemic status. ${ }^{18} 20$ These changes make patients with diabetes more vulnerable to infections, such as foot ulcers and urinary tract infections. ${ }^{2122}$ Moreover, patients with diabetes are prone to infections caused by resistant pathogens. ${ }^{22}$ Mor et al reported that patients with diabetes had a higher rate of hospital-treated infections, with especially increased rates of emphysematous cholecystitis, abscess, tuberculosis and septicaemia. ${ }^{11}$ However, such adverse effects could be reversed after adequate glycaemic control. ${ }^{18}$ 23-27

In our study, DSCP participants had significantly higher prevalence of genitourinary infection and musculoskeletal infection. This phenomenon could be explained by the prevalence of these infectious diseases in patients with type 2 diabetes and the regular follow-up of the DSCP participants. Previous studies showed that patients with diabetes have higher prevalence of genitourinary tract infection and diabetic foot infection. ${ }^{21} 2829$ As the DSCP provides participants with routine laboratory analysis and self-care education, these common infections could be detected earlier and be treated more aggressively. Moreover, given routinely visits and follow-up, the frequencies of infection-related hospitalisation due to respiratory disease, primary bacteraemia and sepsis were significantly decreased in DSCP participants. This might be associated with the regular monitoring and early intervention in the care programme (DSCP). In the logistical regression model, each mortality rate stratified by infection classification was reduced in the DSCP group. In addition, the overall mortality rate was significantly reduced.

Taiwan's NHI programme is a universal health insurance system covering nearly $99 \%$ of its residents. ${ }^{13}$ This programme has been adopted by the Taiwanese government since March 1995. ${ }^{30}$ The revenue for the NHI programme comes from several sources (employees, employers and government, both nationally and locally). ${ }^{30}$ The insured are classified into six main categories and 15 subcategories based on their job and income. The percentage of the premium paid by the insured for each category varies from $0 \%$ for low-income citizens to $100 \%$ for the self-employed. ${ }^{30}$ The DSCP is a programme of care in Taiwan's NHI programme. Our study demonstrated the forest plot (figure 3) for the stratification of ORs of the efficacy of DSCP, finding that DSCP participants with premiums lower than NT $\$ 40000$ benefited more from the DSCP. For the nearly $99 \%$ of citizens covered by Taiwan National Insurance, those with lower incomes may have more accessibility to medical aids. While participating in the DSCP, these individuals could benefit more from receiving diabetes-related education and monitoring during every visit.

This study has some limitations. This study was not a randomised controlled trial, and randomly assigning the patients with diabetes into the DSCP and non-DSCP groups was difficult. In addition, since the physicians were free to recruit their participants, selection bias may also be present. One previous study reported that physicians might exclude sicker patients from this programme. ${ }^{31}$

\section{CONCLUSION}

In this study, we found that participation in the DSCP was associated with a lower risk of hospital mortality in infectious conditions. Older patients, male patients and patients with lower socioeconomic statuses showed higher cost-effectiveness in this programme. This finding may promote healthcare providers distributing this health resource more efficiently.

\section{Author affiliations}

${ }^{1}$ Department of Emergency Medicine, Taipei Veterans General Hospital, Taipei, Taiwan

${ }^{2}$ School of Medicine, National Yang-Ming University, Taipei, Taiwan

${ }^{3}$ YongLin Healthcare Foundation, Taipei, Taiwan

${ }^{4}$ Department of Emergency Medicine, Taichung Veterans General Hospital, Taichung, Taiwan

${ }^{5}$ School of Medicine and Institute of Medicine, Chung Shan Medical University, Taichung, Taiwan

${ }^{6}$ Institute of Occupational Medicine and Industrial Hygiene, National Taiwan University College of Public Health, Taipei, Taiwan

${ }^{7}$ Management Office for Health Data, China Medical University, Taichung, Taiwan ${ }^{8}$ Department of Health Services Administration, China Medical University, Taichung, Taiwan

${ }^{9}$ Department of Emergency Medicine, Taipei Veterans General Hospital, Taoyuan Branch, Taoyuan, Taiwan

Acknowledgements We thank the Biostatistics Task Force of Taichung Veterans General Hospital, Taichung, Taiwan, ROC, for their assistance and advice regarding the statistical analysis.

Contributors Conception and design: $\mathrm{CHC}$ and MSH. Data analysis and interpretation: SYH, DHTY, CKH, CMC, JHC and VCRH. Manuscript writing: $\mathrm{CHC}, \mathrm{MSH}$ and SHM. Final approval and critical revision: CKH and MSH.

Funding This work was supported by grants from the Ministry of Health and Welfare, Taiwan (MOHW107-TDU-B-212-123004); the China Medical University Hospital (DMR-107-192); the Academia Sinica Stroke Biosignature Project (BM10701010021); the MOST Clinical Trial Consortium for Stroke (MOST 106-2321-B-039-005); the Tseng-Lien Lin Foundation; and Taichung, Taiwan, and Katsuzo and Kiyo Aoshima Memorial Funds, Japan.

Competing interests None declared.

Patient consent Not required.

Ethics approval Institutional Review Board of China Medical University (CMUH104-REC2-115).

Provenance and peer review Not commissioned; externally peer reviewed.

Data sharing statement According to the requirement of the International Committee of Medical Journal Editors, we are willing to share the study protocol and statistical analysis process. However, the deidentified data of the patients included in our study were not released to the researchers because of special restriction of the Taiwan National Health Insurance Research Database. After the study is published, the above-mentioned information should be easily available by direct contact with the corresponding author.

Open access This is an open access article distributed in accordance with the Creative Commons Attribution Non Commercial (CC BY-NC 4.0) license, which permits others to distribute, remix, adapt, build upon this work non-commercially, and license their derivative works on different terms, provided the original work is properly cited and the use is non-commercial. See: http://creativecommons.org/ licenses/by-nc/4.0/

(C) Article author(s) (or their employer(s) unless otherwise stated in the text of the article) 2018. All rights reserved. No commercial use is permitted unless otherwise expressly granted. 


\section{REFERENCES}

1. Wang CY, Yu NC, Sheu WH, et al. Team care of type 2 diabetes mellitus in Taiwan. Diabetes Res Clin Pract 2014;106(Suppl 2):S309-13.

2. Bhattarai MD. Three patterns of rising type 2 diabetes prevalence in the world: need to widen the concept of prevention in individuals into control in the community. JNMA J Nepal Med Assoc 2009;48:173-9.

3. Wong KC, Wang Z. Prevalence of type 2 diabetes mellitus of Chinese populations in Mainland China, Hong Kong, and Taiwan. Diabetes Res Clin Pract 2006;73:126-34.

4. Freid VM, Bernstein AB, Bush MA. Multiple chronic conditions among adults aged 45 and over: trends over the past 10 years. NCHS Data Brief 2012:1-8.

5. Hsieh HM, Gu S-M, Shin S-J, et al. Cost-Effectiveness of a Diabetes Pay-For-Performance Program in Diabetes Patients with Multiple Chronic Conditions. PLoS One 2015;10:e0133163.

6. Tsai WL, Yang CY, Lin SF, et al. Impact of obesity on medical problems and quality of life in Taiwan. Am J Epidemiol 2004;160:557-65.

7. Tseng LN, Tseng YH, Jiang YD, et al. Prevalence of hypertension and dyslipidemia and their associations with micro- and macrovascular diseases in patients with diabetes in Taiwan: An analysis of nationwide data for 2000-2009. J Formos Med Assoc 2012;111:625-36.

8. Tan EC, Pwu RF, Chen DR, et al. Is a diabetes pay-for-performance program cost-effective under the National Health Insurance in Taiwan? Qual Life Res 2014;23:687-96.

9. Hao LJ, Tien KJ, Chao H, et al. Metabolic outcome for diabetes shared care program outpatients in a veterans hospital of southern Taiwan. J Chin Med Assoc 2011;74:287-93.

10. Liu Z, Fu C, Wang W, et al. Prevalence of chronic complications of type 2 diabetes mellitus in outpatients - a cross-sectional hospital based survey in urban China. Health Qual Life Outcomes 2010;8:62.

11. Mor A, Berencsi K, Nielsen JS, et al. Rates of Community-based Antibiotic Prescriptions and Hospital-treated Infections in Individuals With and Without Type 2 Diabetes: A Danish Nationwide Cohort Study, 2004-2012. Clinical Infectious Diseases 2016;63:501-11.

12. Kornelius E, Chiou JY, Yang YS, et al. The Diabetes Shared Care Program and Risks of Cardiovascular Events in Type 2 Diabetes. Am $J$ Med 2015;128:977-85.

13. Lee TT, Cheng SH, Chen CC, et al. A pay-for-performance program for diabetes care in Taiwan: a preliminary assessment. Am J Manag Care 2010;16:65-9.

14. Rosenbaum PR, Rubin DB. The central role of the propensity score in observational studies for causal effects. Biometrika 1983;70:41-55.

15. Austin PC. An Introduction to Propensity Score Methods for Reducing the Effects of Confounding in Observational Studies. Multivariate Behav Res 2011;46:399-424.
16. Chen PC, Lee YC, Kuo RN. Differences in patient reports on the quality of care in a diabetes pay-for-performance program between 1 year enrolled and newly enrolled patients. Int J Qual Health Care 2012;24:189-96.

17. Delamaire M, Maugendre D, Moreno M, et al. Impaired leucocyte functions in diabetic patients. Diabet Med 1997;14:29-34.

18. Shilling AM, Raphael J. Diabetes, hyperglycemia, and infections. Best Pract Res Clin Anaesthesiol 2008;22:519-35.

19. Aragon D, Ring CA, Covelli M. The influence of diabetes mellitus on postoperative infections. Crit Care Nurs Clin North Am 2003;15:125-35.

20. McManus LM, Bloodworth RC, Prihoda TJ, et al. Agonist-dependent failure of neutrophil function in diabetes correlates with extent of hyperglycemia. J Leukoc Biol 2001;70:395-404.

21. Huang YY, Lin KD, Jiang YD, et al. Diabetes-related kidney, eye, and foot disease in Taiwan: an analysis of the nationwide data for 2000 2009. J Formos Med Assoc 2012;111:637-44.

22. Nitzan O, Elias M, Chazan B, et al. Urinary tract infections in patients with type 2 diabetes mellitus: review of prevalence, diagnosis, and management. Diabetes Metab Syndr Obes 2015;8:129-36.

23. Mowat A, Baum J. Chemotaxis of polymorphonuclear leukocytes from patients with diabetes mellitus. N Engl J Med 1971;284:621-7.

24. Bagdade JD, Root RK, Bulger RJ. Impaired leukocyte function in patients with poorly controlled diabetes. Diabetes 1974;23:9-15.

25. MacRury SM, Gemmell CG, Paterson KR, et al. Changes in phagocytic function with glycaemic control in diabetic patients. $J$ Clin Pathol 1989;42:1143-7.

26. Bagdade JD, Stewart M, Walters E. Impaired granulocyte adherence. A reversible defect in host defense in patients with poorly controlled diabetes. Diabetes 1978;27:677-81.

27. Bagdade JD, Walters E. Impaired granulocyte adherence in mildly diabetic patients: effects of tolazamide treatment. Diabetes 1980;29:309-11.

28. Tseng $\mathrm{CH}$. Prevalence and risk factors of diabetic foot problems in Taiwan: a cross-sectional survey of non-type 1 diabetic patients from a nationally representative sample. Diabetes Care 2003;26:3351.

29. Margolis DJ, Malay DS, Hoffstad OJ, et al. Prevalence of diabetes, diabetic foot ulcer, and lower extremity amputation among Medicare beneficiaries, 2006 to 2008: Data Points \#1. Data Points Publication Series. Rockville (MD): Agency for Healthcare Research and Quality (US), 2011

30. Wu TY, Majeed A, Kuo KN. An overview of the healthcare system in Taiwan. London J Prim Care 2010;3:115-9.

31. Chang RE, Lin SP, Aron DC. A pay-for-performance program in Taiwan improved care for some diabetes patients, but doctors may have excluded sicker ones. Health Aff 2012;31:93-102. 